\title{
Saúde bucal e índice de desenvolvimento humano, Bahia, 2001-2014
}

\section{Oral health and human development index, Bahia, Brazil, 2001-2014}

\section{Índice de salud oral y desarrollo humano, Bahia, Brasil, 2001-2014}

\author{
Arine Sales dos Santos ${ }^{1}$ \\ Sandra Garrido Barros ${ }^{2}$ \\ Denise Nogueira Cruz ${ }^{3}$ \\ Maria Cristina Teixeira Cangussu
}

RESUMO: Estudo ecológico de série histórica que avaliou indicadores de saúde bucal em relação a cobertura da Estratégia Saúde da Família (ESF), o porte populacional e Índice de Desenvolvimento Humano Municipal (IDH-M), entre 2001-2014, nos municípios da Bahia. As informações foram obtidas nas bases de dados do DATASUS e IBGE. Inicialmente, procedeu-se a compatibilização das informações das diferentes bases de dados e cálculo dos indicadores. A análise foi realizada através do Minitab 17, utilizando-se o teste de Pearson para correlação com um nível de significância de 5\%. Observou-se consistência no registro de informações ambulatoriais e aumento das coberturas da ESF e de Equipes de Saúde Bucal (ESB), não proporcional à cobertura de $1^{a}$ consulta odontológica, que apresentou crescimento menor até 2012, seguido de declínio. A média de escovação supervisionada apresentou grande variação. Entre 2001 e 2007, a média de procedimentos básicos apresentou crescimento, enquanto as ações especializadas, apesar do aumento até 2005, caíram em 2006 e 2007. A análise de correlação evidenciou associação positiva entre porte populacional e coberturas da ESF e de ESB (menor porte populacional, maior cobertura), assim como entre IDH-M e estes indicadores (menor IDH-M, maior cobertura). Na Bahia, a implantação da ESF e das ESB tem representado um grande desafio para os municípios de grande porte populacional e melhor IDH.

Palavras-chave: Indicadores de saúde bucal. Estratégia de Saúde da Família. Saúde Bucal.

ABSTRACT: Historical series ccological study that evaluated oral health indicators in relation to the estimated coverage of Family Health Strategy (FHS), population size and Municipal Human Development Index (HDI), from 2001 to 2014 in municipalities of Bahia, Brazil. The data was obtained from health information systems maintained by the Department of Informatics of SUS

1 Cirurgiã-dentista. Residente em Saúde da Família pela Fundação Estatal de Saúde da Família - Bahia.

2 Doutora em Saúde Pública. Professora Adjunta do Departamento de Odontologia Social e Pediátrica/ UFBA

3 Doutora em Saúde Pública. Professora Adjunta do Departamento de Odontologia Social e Pediátrica/ UFBA

ISSN 1982-8829 Tempus, actas de saúde colet, Brasília, 13(3), 25-42, set, 2019. Epub Jul/2020 
(DATASUS) and of the Brazilian Institute of Geography and Statistics (IBGE). The collected data were organized in electronic spreadsheets to make compatible the information of different databases and calculation of indicators. Analysis was performed in Minitab 17 statistical package and the Pearson test was used for correlation with significance level of 5\%. It was observed consistency in the information record in the SIA/SUS for the analyzed period and a significant increase in the coverage of the FHS and Oral Health Teams (OHT), that was not proportional to that observed to coverage of 1st dental consultation, smaller until 2012, and followed by a decline. The average of supervised brushing had great variation. Between 2001 and 2007, the average number of basic procedures presented growth while the average number of specialized actions, despite the significant increase until 2005, fell dramatically in 2006 and 2007. The correlation analysis showed positive association between population size and coverage of the FHS and OHT (lower population size, higher coverage), as well as between HDI-M and these indicators (lower HDI-M, higher coverage). In Bahia, implementation of FHS and OHT has been a major challenge for municipalities with large populations and better HDI.

Key words: Oral health indicators. Family Health Strategy. Oral Health.

RESUMEN: Estudio ecológico de series históricas que evaluó los indicadores de salud bucal en relación con la cobertura de la Estrategia de Salud de la Familia (FHS), el tamaño de la población y el Índice de Desarrollo Humano Municipal (IDH-M), entre 2001-2014, en los municipios de Bahía. La información se obtuvo de las bases de datos DATASUS e IBGE. Inicialmente, la información de las diferentes bases de datos se hizo compatible y se calcularon los indicadores. El análisis se realizó con Minitab 17, con la prueba de correlación de Pearson con un nivel de significación del 5\%. Se observó consistencia en el registro de información ambulatoria y un aumento en la cobertura de los Equipos de Salud Oral y FHS (BHS), no proporcional a la cobertura de la primera cita dental, que mostró un menor crecimiento hasta 2012, seguido de una disminución. El cepillado medio supervisado varió ampliamente. Entre 2001 y 2007, el promedio de los procedimientos básicos aumentó, mientras que las acciones especializadas, a pesar del aumento hasta 2005, cayeron en 2006 y 2007. El análisis de correlación mostró una asociación positiva entre el tamaño de la población y la cobertura de ESF y ESB (tamaño más pequeño). así como entre HDI-M y estos indicadores (menor HDI-M, mayor cobertura). En Bahía, la implementación del FSE y el ESB ha sido un gran desafío para los municipios de gran población y un mejor IDH.

Palabras clave: Indicadores de salud bucal. Estrategia de Salud de la Familia. Salud bucal.

\section{INTRODUÇÃO}

O conceito de políticas de saúde envolve intervenções organizadas pelo Estado através de diferentes governos, com a participação de agentes e instituições governamentais e não governamentais, objetivando enfrentamento de situações-problema identificadas como necessidades sociais por diferentes grupos ${ }^{1}$. Nesse sentido, desde a criação do Sistema Único de Saúde (SUS) diversas políticas de saúde vêm sendo implementadas atendendo às demandas da sociedade.

Tempus, actas de saúde colet, Brasília, 13(3), 25-42, set, 2019. Epub Jul/2020 ISSN 1982-8829 
A descentralização das ações de saúde no Brasil durante os anos 1990 trouxe a necessidade de monitoramento mais frequente, o que levou à formulação dos pactos de indicadores. Segundo o Ministério da Saúde², o Pacto foi firmado tendo como metas anuais alcançar determinados valores dos indicadores de saúde acordados, bem como organizar a oferta de serviços. Desde 1999 tenta-se ampliar o monitoramento da aplicação dos recursos e do impacto na saúde da população ${ }^{3}$. As Portarias $n^{\circ} 3.925$ de 1998 e $n^{\circ} 476$ de 1999 criaram o Pacto de Indicadores da Atenção Básica (PIAB), constituído como instrumento formal de negociação das três esferas de gestão (União, Estados e Municípios), com a pactuação de metas para indicadores previamente acordados, indicando a intenção dos gestores de induzir melhorias na atenção básica e na saúde da população.

A Estratégia Saúde da Família (ESF), lançada pelo Ministério da Saúde em 1994, com caráter substitutivo ao modelo hegemônico foi orientada pelos princípios de adscrição de clientela, territorialização, diagnóstico da situação de saúde da população e planejamento local ${ }^{4}$. Esses princípios traduzem-se em um novo vínculo entre as equipes de saúde e as unidades, definição do território e da população a ser trabalhada, análise da situação de saúde, planejamento com bases na situação do local, bem como atividades segundo classificação de risco da população ${ }^{2,5}$. Constituiuse então como estratégia prioritária para organização da atenção primária, pautada nos princípios do SUS. De modo que possibilita o acesso aos serviços de saúde e estabelece uma relação de vínculo com a sociedade ${ }^{2}$. Naquele momento, cada Equipe de Saúde da Família (EqSF) deveria ser composta por um médico generalista, um enfermeiro, auxiliares de enfermagem e agentes comunitários de saúde e faria a cobertura de uma área onde residissem no máximo 1.000 famílias ou cerca de 4.500 pessoas $^{5}$. Os profissionais de saúde bucal não faziam parte do programa.

No final do ano 2000, o Ministério da Saúde criou o incentivo financeiro para a inclusão de equipes de saúde bucal na Estratégia Saúde da Família e, a partir de 2003, a saúde bucal em todos os níveis de atenção foi reorganizada passando a ocupar lugar de destaque no governo federal ${ }^{6}$.

A tomada de decisão por parte de profissionais de saúde e gestores deve ser subsidiada por informações acerca das condições do estado de saúde da população de abrangência e sobre os serviços de saúde existentes em determinada área. Os indicadores do estado de saúde bucal representam o quadro epidemiológico acerca dos principais agravos. Nesse sentido, o Ministério da Saúde tem realizado regularmente inquéritos de abrangência nacional para acompanhamento dos principais agravos de saúde bucal. Os indicadores de serviços podem ser relativos a rede instalada e produção ambulatorial, como cobertura estimada das equipes de saúde bucal, cobertura de primeira consulta odontológica, média anual de escovação supervisionada, média de ações básicas e especializadas, proporção de exodontias em relação às ações odontológicas básicas.

Os indicadores de saúde constituem uma importante ferramenta para avalição dos recursos destinados a atenção da saúde, uma vez que, possibilitam uma avaliação do impacto da aplicação destes recursos na população. O Sistema de Informações Ambulatoriais do Sistema Único de 
Saúde (SIA/SUS) consolida as informações sobre produção ambulatorial, sendo a principal fonte para cálculo dos indicadores de produção de serviços de saúde bucal. É uma importante base para o acompanhamento e a avaliação dos serviços de saúde, sendo possível, a partir da construção de séries históricas de indicadores de serviço, caracterizar a prática odontológica e orientar a avaliação da organização da saúde bucal nos municípios ${ }^{7,8}$.

Alguns estudos mostram que os indicadores de saúde bucal são instrumentos importantes na avalição dos recursos destinados a essa área na atenção básica, devendo ser considerados na implementação de políticas públicas de forma equânime ${ }^{9}$. Estudo por meio do uso de sistemas de informação verificou a existência de correlação entre indicadores da atenção primária em saúde bucal e os de desenvolvimento humano, verificando que os sistemas de informação se constituem importantes ferramentas no planejamento local das ações de saúde bucal dos municípios ${ }^{10}$.

Alguns estudos avaliaram os serviços públicos e a gestão da política pública de saúde bucal a partir da análise de indicadores de produção ambulatorial obtidos por meio de dados secundários ${ }^{3,}$ 7,11-14 e, dentre esses, apenas três ${ }^{3,13,14}$ analisaram a relação entre a provisão de serviços e o Índice de Desenvolvimento Humano Municipal (IDH-M). Geralmente, os estudos sobre indicadores de saúde bucal são específicos para cada Unidade Federativa ${ }^{3}$.

Scarparo, Ditterich ${ }^{15}$ realizaram um estudo sobre o impacto da atual Política Nacional de Saúde Bucal na provisão de serviços odontológicos do Estado do Rio de Janeiro, concluindo que a mesma favoreceu a organização dos serviços de saúde bucal nos municípios fluminenses. Os principais desafios seriam a necessidade de aumentar a cobertura das equipes, pensar a Política Nacional de Saúde Bucal de forma regionalizada e articulada com as demais políticas públicas, propiciando o desenvolvimento de ações intersetoriais, a superação das desigualdades sociais e o maior acesso às ações e serviços de saúde de forma integral ${ }^{15}$. Outro desafio é que o modelo assistencial tradicional, centrado nas Unidades Básicas de Saúde, ainda mostra melhores resultados em relação a alguns indicadores de saúde bucal no serviço público em alguns municípios ${ }^{16,17}$.

Os estudos que analisam indicadores de produção ambulatorial em saúde bucal são restritos a determinadas Unidades Federativas ${ }^{10,15,17,18}$ ou região metropolitana ${ }^{14}$. Os estudos que propõem uma abordagem de âmbito nacional têm por base as unidades federativas sem análises desagregadas por municípios ${ }^{3}$.

O presente estudo teve por objetivo analisar o comportamento dos indicadores de saúde bucal no período de 2001 a 2014 nos municípios do estado da Bahia e sua relação com o porte populacional e o Índice de Desenvolvimento Humano Municipal (IDH-M). 


\section{METODOLOGIA}

Foi realizado um estudo de série histórica de indicadores de saúde bucal dos 417 municípios do estado da Bahia, a partir de dados obtidos nos sistemas de informação em saúde mantidos pelo Departamento de Informática do SUS (DATASUS) para o período de 2001 a 2014 . Foram analisados os seguintes indicadores:

Quadro 1 - Indicadores de Saúde Bucal e forma de cálculo

\begin{tabular}{|c|c|}
\hline INDICADORES & FORMA DE CÁLCULO \\
\hline Cobertura estimada de ESB & $\begin{array}{l}\text { Número de ESF x } 3450 \\
\text { População }\end{array}$ \\
\hline $\begin{array}{l}\text { Cobertura da } 1^{\mathrm{a}} \text { consulta } \\
\text { odontológica (código 03.021.01-7) }\end{array}$ & $\begin{array}{c}\text { Número de } 1^{\mathrm{a}} \text { consulta odontológica } \\
\text { População }\end{array}$ \\
\hline $\begin{array}{l}\text { Cobertura de ação coletiva de } \\
\text { escovação supervisionada (código } \\
\text { SIA-SUS 01.01.02.003-1) }\end{array}$ & $\begin{array}{l}\text { Número de pessoas participantes de } \\
\text { escovação supervisionada/12meses } \\
\text { Populacão X } 100\end{array}$ \\
\hline Média de ações básicas individuais & $\begin{array}{l}\text { Número de procedimentos odontológicos básicos } \\
\text { Populacão }\end{array}$ \\
\hline Média de ações especializadas & $\begin{array}{c}\text { Número de procedimentos odontológicos } \\
\text { especializados } \\
\text { População }\end{array}$ \\
\hline
\end{tabular}

As médias anuais de escovação supervisionada; média de ações básicas e especializadas; e a cobertura de primeira consulta odontológica são indicadores que se referem à produção ambulatorial e disponibilizados pelo SIA/SUS. Os dados foram coletados a partir do endereço eletrônico do DATASUS (http://datasus.saude.gov.br), através do aplicativo Tabnet, e do portal do Departamento de Atenção Básica do Ministério da Saúde (DAB/MS). Para as informações sociodemográficas foi consultado o portal do IBGE (http://www.ibge.gov.br). A coleta foi realizada no período de julho a outubro de 2015. Os dados foram organizados em planilhas eletrônicas no programa Microsoft Excel para compatibilização das informações oriundas das diferentes bases consultadas. Esse procedimento foi realizado por dois pesquisadores diferentes (dupla entrada) para que fosse efetuada na sequência, e através do pacote estatístico Minitab 17, a verificação e análise dos dados obtidos por cada pesquisador de modo a identificar potenciais equívocos. Não foram encontradas discrepâncias entre os dados. Os indicadores de saúde bucal foram analisados em relação à cobertura estimada da Estratégia Saúde da Família (ESF), ao porte populacional e ao IDH-M no período de 2001 a 2014. Foi utilizado para a análise de correlação o teste de Pearson. 


\section{RESULTADOS}

Foram incluídos no estudo os 417 municípios do estado da Bahia. A cobertura de primeira consulta odontológica teve crescimento no período analisado, sendo que nos anos de 2007, 2009, 2013 e 2014 observou-se um decréscimo. Vale acrescentar que nos anos de 2011 e 2012 houve um crescimento significativo, com destaque para o ano de 2012 que apresentou a maior média no período estudado. Para todos os indicadores de serviço, notou-se melhora significativa do percentual de municípios com registro de informações de procedimentos no SIA-SUS entre 2001 e 2014 (Tabela 1).

Tabela 1 - Cobertura média de $1^{\text {a }}$ Consulta Odontológica, Escovação Supervisionada, Procedimentos de Ações Básicas (2001-2007), Procedimentos de Ações Especializadas (20012007), Equipe de Saúde da Família, Equipe da Saúde Bucal e percentual de municípios sem informação, no período de 2001-2014 nos Municípios Baianos.

\begin{tabular}{|c|c|c|c|c|c|c|c|c|c|c|c|c|}
\hline \multirow{2}{*}{ Ano } & \multicolumn{2}{|c|}{$1^{\mathrm{a}}$ consulta } & \multicolumn{2}{|c|}{$\begin{array}{c}\text { Escovação } \\
\text { Supervisionada }\end{array}$} & \multicolumn{2}{|c|}{ Ações básicas } & \multicolumn{2}{|c|}{$\begin{array}{c}\text { Ações } \\
\text { especializadas }\end{array}$} & \multicolumn{2}{|c|}{ Cobertura ESF } & \multicolumn{2}{|c|}{$\begin{array}{l}\text { Cobertura } \\
\text { ESB }\end{array}$} \\
\hline & Média & $\begin{array}{c}\% \\
\text { S.I. }\end{array}$ & Média & $\%$ S.I. & Média & $\%$ S.I. & Média & $\%$ S.I. & Média & $\begin{array}{l}\% \\
\text { S.I. }\end{array}$ & Média & $\begin{array}{c}\% \\
\text { S.I. }\end{array}$ \\
\hline 2001 & 9,5 & 21,8 & 0,01 & 53,2 & 0,4 & 12 & 0,1 & 85,1 & 17,1 & - & 2,9 & - \\
\hline 2002 & 10,8 & 17,5 & 0,03 & 41,2 & 0,7 & 8,6 & 0,1 & 85,9 & 26,1 & - & 6,8 & - \\
\hline 2003 & 12 & 14,4 & 0,03 & 38,6 & 0,7 & 7 & 0,1 & 84,2 & 32,8 & - & 11,3 & - \\
\hline 2004 & 12,4 & 13,4 & 0,03 & 40,8 & 0,7 & 8,6 & 0,1 & 83,9 & 38,8 & $\begin{array}{l}- \\
-\end{array}$ & 16,7 & - \\
\hline 2005 & 13,3 & 9,6 & 0,02 & 34,5 & 0,7 & 6,5 & 0,4 & 75,5 & 59,6 & $\begin{array}{l}- \\
-\end{array}$ & 30,5 & $\begin{array}{l}- \\
-\end{array}$ \\
\hline 2006 & 17,4 & 8,9 & 0,01 & 0 & 0,9 & 5 & 0 & 74,1 & 68,6 & $\begin{array}{l}- \\
-\end{array}$ & 42,6 & $\begin{array}{l}- \\
-\end{array}$ \\
\hline 2007 & 16,8 & 7,9 & 0,02 & 53,5 & 1,0 & 4,8 & 0,1 & 78,4 & 66,1 & - & 37,6 & $\begin{array}{l}- \\
-\end{array}$ \\
\hline 2008 & 18,6 & 8,2 & 0,02 & 32,4 & - & 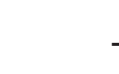 & - & - & 72,4 & - & 45,3 & $\begin{array}{l}- \\
-\end{array}$ \\
\hline 2009 & 17,2 & 6 & 0,02 & 21,3 & - & . & - & . & 76,7 & $\begin{array}{l}- \\
-\end{array}$ & 51,8 & - \\
\hline 2010 & 18,2 & 5,5 & 0,02 & 20,6 & - & - & - & . & 83,7 & - & 58,3 & - \\
\hline 2011 & 31,1 & 5,8 & 0,02 & 18 & - & . & - & 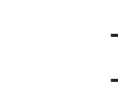 & 87 & $\begin{array}{l}- \\
-\end{array}$ & 61,9 & $\begin{array}{l}- \\
-\end{array}$ \\
\hline 2012 & 58,1 & 6,2 & 0,12 & 22,1 & - & & - & . & 88,9 & - & 63,7 & - \\
\hline 2013 & 25 & 4,1 & 0,01 & 20,9 & - & . & - & 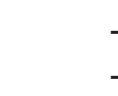 & 87 & $\begin{array}{l}- \\
-\end{array}$ & 64,6 & - \\
\hline 2014 & 21,1 & 3,8 & 0,01 & 21,3 & - & 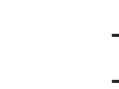 & - & 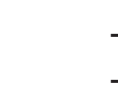 & 96 & - & 66,9 & - \\
\hline
\end{tabular}

${ }^{1}$ A partir do ano de 2008, houve uma mudança na tabela do SIA/SUS que dificultou a obtenção das informações Tempus, actas de saúde colet, Brasília, 13(3), 25-42, set, 2019. Epub Jul/2020 ISSN 1982-8829 


\section{$31 / /$}

de Ações Básicas e Ações Especializadas em Saúde Bucal. \% S.I.: percentual de municípios sem informação registrada no Sistema de Informação Ambulatorial.

Com relação à cobertura da ação coletiva de escovação supervisionada, foi grande a quantidade de municípios que não foram identificadas as informações das suas produções, o que pode justificar o crescimento insignificante da mesma. Notou-se que, mesmo nos anos com menor ausência de informações, o indicador manteve-se menor ou igual aos períodos com maior número de municípios sem informação, como visto no ano de 2006 em que todos os municípios informaram a quantidade de escovações supervisionadas realizadas e, entretanto, o respectivo indicador revelou uma média igual aos anos de 2013 (20,9\% dos municípios sem informação) e 2014 (21,3\% dos municípios sem informação) (Tabela 1).

De 2001 a 2007 a média de Ações Odontológicas Básicas apresentou tendência de crescimento, bem como os municípios que informaram esses dados. Já para a média de ações odontológicas especializadas, a tendência foi de manutenção, apresentando nos anos de 2001, 2002, 2003, 2004 e 2007 médias próximas de 0,10, com exceções nos anos de 2005 (média de 0,40) e o ano de 2006 (média menor que 0,10). Grande parte dos municípios baianos não produziram informações referentes à realização de ações odontológicas especializadas durante esse período, com percentual de municípios sem registro de informação variando entre $74,1 \%$ a $85,9 \%$ (Tabela 1 ).

Observou-se aumento importante na cobertura da Estratégia Saúde da Família no período analisado, sendo que nos anos 2007 e 2013 ocorreu um decréscimo pouco significativo. A cobertura de Equipe de Saúde Bucal também apresentou aumento, com diminuição apenas no ano de 2007, também pouco significativa. Vale acrescentar que todos os municípios apresentavam dados de Equipe de Saúde da Família e Equipe de Saúde Bucal, obtidos juntos ao Departamento de Atenção Básica do Ministério da Saúde (Tabela 1).

Os indicadores de cobertura da Estratégia Saúde da Família e cobertura de Equipes de Saúde Bucal, segundo porte populacional, no período de 2001-2014 revelaram que quanto menor o porte populacional, maior a população coberta pela ESF, sendo essa diferença estatisticamente significante $(\mathrm{P}<0,05)$ para todo o período, exceto em $2002(\mathrm{P}=0,06)$. Para a cobertura de Equipes de Saúde Bucal, observou-se tendência semelhante, sendo a diferença também estatisticamente significante $(\mathrm{P}<0,05)$ a partir do ano de 2005. (Tabela 2) 
Tabela 2- Cobertura média de Equipe da Saúde da Família, Equipe de Saúde Bucal, por Porte Populacional no período de 2001-2014, nos Municípios Baianos.

\begin{tabular}{|c|c|c|c|c|c|c|c|c|c|}
\hline \multirow{2}{*}{ Ano } & & \multicolumn{6}{|c|}{ Porte populacional } & \multicolumn{2}{|c|}{$\mathrm{P}$ valor } \\
\hline & & $\begin{array}{c}>900.00 \\
\text { hab. }\end{array}$ & $\begin{array}{c}500.001 \\
-900.000 \\
\text { hab. }\end{array}$ & $\begin{array}{c}100.001- \\
500.000 \\
\text { hab. }\end{array}$ & $\begin{array}{c}50.001- \\
100.000 \\
\text { hab. }\end{array}$ & $\begin{array}{c}20.001- \\
50.000 \\
\text { hab. }\end{array}$ & $\begin{array}{c}<20.000 \\
\text { hab. }\end{array}$ & ESF & ESB \\
\hline \multirow[t]{3}{*}{2001} & $\mathrm{~N}$ & 1 & 1 & 15 & 31 & 132 & 237 & & \\
\hline & ESF & 1,7 & 12,9 & 20,3 & 16,1 & 15,2 & 18,2 & 0,04 & 0,97 \\
\hline & ESB & 0,5 & 0 & 5 & 3,1 & 2,7 & 2,8 & & \\
\hline \multirow{3}{*}{2002} & $\mathrm{~N}$ & 1 & 1 & 15 & 31 & 132 & 237 & & \\
\hline & ESF & 2,4 & 15,1 & 27 & 19,5 & 22,9 & 28,7 & 0,06 & 0,77 \\
\hline & ESB & 0,8 & 0 & 6,5 & 4,6 & 5,7 & 7,7 & & \\
\hline \multirow[t]{3}{*}{2003} & $\mathrm{~N}$ & 1 & 1 & 15 & 31 & 132 & 237 & & \\
\hline & ESF & 3,6 & 22 & 31,7 & 22,9 & 29,8 & 36 & 0,01 & 0,56 \\
\hline & ESB & 1,3 & 0 & 10,4 & 8,3 & 9,2 & 13 & & \\
\hline \multirow[t]{3}{*}{2004} & $\mathrm{~N}$ & 1 & 1 & 15 & 31 & 132 & 237 & & \\
\hline & ESF & 9,4 & 35,2 & 34,6 & 24,7 & 31,5 & 45 & 0,01 & 0,25 \\
\hline & ESB & 7,2 & 6,5 & 16 & 13,7 & 12,5 & 19,5 & & \\
\hline \multirow[t]{3}{*}{2005} & $\mathrm{~N}$ & 1 & 1 & 15 & 31 & 132 & 237 & & \\
\hline & ESF & 12,3 & 40,9 & 37,3 & 36 & 50 & 69,7 & 0,00 & 0,01 \\
\hline & ESB & 8,8 & 6,3 & 20,2 & 16,3 & 26 & 35,8 & & \\
\hline \multirow[t]{3}{*}{2006} & $\mathrm{~N}$ & 1 & 1 & 15 & 31 & 132 & 237 & & \\
\hline & ESF & 12,7 & 39,7 & 45,6 & 42,3 & 56,9 & 80,4 & 0,00 & 0,00 \\
\hline & ESB & 9,4 & 10,6 & 27,5 & 25,9 & 35,1 & 50,2 & & \\
\hline \multirow[t]{3}{*}{2007} & $\mathrm{~N}$ & 1 & 1 & 15 & 31 & 132 & 237 & & \\
\hline & ESF & 12,5 & 39,7 & 44 & 42,1 & 58,3 & 75,4 & 0,00 & 0,03 \\
\hline & ESB & 9 & 11 & 26,8 & 24,9 & 33,6 & 42,4 & & \\
\hline \multirow[t]{3}{*}{2008} & $\mathrm{~N}$ & 1 & 1 & 15 & 31 & 132 & 237 & & \\
\hline & ESF & 8,1 & 41,6 & 48,7 & 48,3 & 65,4 & 81,4 & 0,00 & 0,00 \\
\hline & ESB & 5,7 & 14,9 & 34,1 & 29,4 & 40,4 & 51,1 & & \\
\hline \multirow[t]{3}{*}{2009} & $\mathrm{~N}$ & 1 & 1 & 15 & 31 & 132 & 237 & & \\
\hline & ESF & 9,1 & 42,2 & 47,2 & 46,9 & 68,4 & 87,5 & 0,00 & 0,00 \\
\hline & ESB & 6,6 & 16,3 & 31,8 & 31 & 44,9 & 59,9 & & \\
\hline \multirow[t]{3}{*}{2010} & $\mathrm{~N}$ & 1 & 1 & 15 & 31 & 132 & 237 & & \\
\hline & ESF & 17 & 44,2 & 49,2 & 49,7 & 72,6 & 96,9 & 0,00 & 0,00 \\
\hline & ESB & 10,4 & 17,3 & 34,5 & 34,4 & 48,9 & 68,6 & & \\
\hline \multirow[t]{3}{*}{2011} & $\mathrm{~N}$ & 1 & 1 & 15 & 31 & 132 & 237 & & \\
\hline & ESF & 15,2 & 44,3 & 48,7 & 52,8 & 74,5 & 101,3 & 0,00 & 0,00 \\
\hline & ESB & 10,3 & 17,6 & 34,9 & 37,5 & 50,9 & 73,4 & & \\
\hline \multirow[t]{3}{*}{2012} & $\mathrm{~N}$ & 1 & 1 & 15 & 31 & 132 & 237 & & \\
\hline & ESF & 11,5 & 45,4 & 52 & 56,7 & 76,6 & 102,8 & 0,00 & 0,00 \\
\hline & ESB & 7,8 & 18,5 & 36,9 & 39,3 & 53,1 & 75 & & \\
\hline \multirow[t]{3}{*}{2013} & $\mathrm{~N}$ & 1 & 1 & 15 & 31 & 132 & 237 & & \\
\hline & ESF & 18,6 & 42,6 & 49 & 53,8 & 76,8 & 99,9 & 0,00 & 0,00 \\
\hline & ESB & 10,2 & 18,3 & 34,3 & 37,1 & 52,1 & 77,5 & & \\
\hline \multirow[t]{3}{*}{2014} & $\mathrm{~N}$ & 1 & 1 & 15 & 31 & 132 & 237 & & \\
\hline & ESF & 21,4 & 43,6 & 50,9 & 65,1 & 84 & 110 & 0,00 & 0,00 \\
\hline & ESB & 11 & 18,1 & 34,8 & 38,6 & 55,2 & 79,5 & & \\
\hline
\end{tabular}


Tabela 3 - Cobertura média de Ações Odontológicas Básicas (AB) e Especializadas (AE) segundo Índice de Desenvolvimento Humano Municipal (IDH-M) nos anos de 2001-2014, nos Municípios Baianos.

\begin{tabular}{|c|c|c|c|c|c|c|}
\hline \multirow{2}{*}{ Ano } & & \multicolumn{3}{|c|}{ IDH-M } & \multicolumn{2}{|c|}{$\mathrm{P}$ valor } \\
\hline & & $0,70-0,79$ & $0,55-0,69$ & $\leq 0,54$ & $\mathrm{AB}$ & $\mathrm{AE}$ \\
\hline \multirow[t]{4}{*}{2001} & $\mathrm{~N}$ & 8 & 317 & 42 & \multirow{4}{*}{0,02} & \multirow{4}{*}{0,4} \\
\hline & $\mathrm{AB}$ & 0,59 & 0,46 & 0,25 & & \\
\hline & $\mathrm{N}$ & 6 & 54 & 2 & & \\
\hline & $\mathrm{AE}$ & 0,02 & 0,09 & 1,51 & & \\
\hline \multirow[t]{4}{*}{2002} & $\mathrm{~N}$ & 8 & 327 & 46 & \multirow{4}{*}{0,2} & \multirow{4}{*}{0,64} \\
\hline & $\mathrm{AB}$ & 0,71 & 0,69 & 0,45 & & \\
\hline & $\mathrm{N}$ & 5 & 52 & 2 & & \\
\hline & $\mathrm{AE}$ & 0,03 & 0,1 & 0,14 & & \\
\hline \multirow[t]{4}{*}{2003} & $\mathrm{~N}$ & 8 & 334 & 46 & \multirow{4}{*}{0,03} & \multirow{4}{*}{0,52} \\
\hline & $\mathrm{AB}$ & 0,79 & 0,72 & 0,36 & & \\
\hline & $\mathrm{N}$ & 7 & 56 & 3 & & \\
\hline & $\mathrm{AE}$ & 0,02 & 0,09 & 0,07 & & \\
\hline \multirow[t]{3}{*}{2004} & $\mathrm{~N}$ & 8 & 330 & 43 & \multirow{4}{*}{0,02} & \multirow{4}{*}{0,54} \\
\hline & $\mathrm{AB}$ & 0,96 & 0,73 & 0,38 & & \\
\hline & $\mathrm{N}$ & 6 & 59 & 2 & & \\
\hline \multirow{4}{*}{2005} & ESB & 0,02 & 0,07 & 0,04 & & \\
\hline & $\mathrm{N}$ & 8 & 339 & 43 & \multirow{4}{*}{0,03} & \multirow{4}{*}{0,45} \\
\hline & $\mathrm{AB}$ & 0,98 & 0,75 & 0,43 & & \\
\hline & $\mathrm{N}$ & 7 & 84 & 11 & & \\
\hline \multirow{4}{*}{2006} & ESB & 0,03 & 0,04 & 0,01 & & \\
\hline & $\mathrm{N}$ & 8 & 344 & 44 & \multirow{4}{*}{0,00} & \multirow{4}{*}{0,50} \\
\hline & $\mathrm{AB}$ & 1,54 & 0,99 & 0,46 & & \\
\hline & $\mathrm{N}$ & 7 & 90 & 11 & & \\
\hline \multirow{5}{*}{2007} & $\mathrm{AE}$ & 0,04 & 0,04 & 0,02 & & \\
\hline & $\mathrm{N}$ & 8 & 342 & 47 & \multirow{4}{*}{0,02} & \multirow{4}{*}{0,7} \\
\hline & $\mathrm{AB}$ & 1,28 & 0,99 & 0,57 & & \\
\hline & $\mathrm{N}$ & 8 & 77 & 5 & & \\
\hline & ESB & 0,04 & 0,06 & 0,03 & & \\
\hline
\end{tabular}

É possível perceber na tabela 3, que houve correlação positiva da média de ações odontológicas básicas em relação ao IDH-M, esta foi maior quanto melhor o IDH-M $(\mathrm{P}<0,05)$, exceto em 2002 $(\mathrm{P}=0,20)$. Essa correlação não foi positiva em relação às ações especializadas $(\mathrm{P}>0,05)$. Com relação ao porte populacional, a média de ações básicas não apresentou valores estatisticamente significantes, enquanto a média das ações especializadas apresentou correlação positiva estatisticamente significante apenas no ano de 2001.

Com relação às coberturas da Estratégia Saúde da Família e de Equipes de Saúde Bucal, segundo Índice de Desenvolvimento Humano Municipal no período de 2001-2014, observou-se correlação negativa, ou seja, quanto menor o IDH-M, maior a cobertura populacional, sendo as diferenças 
estatisticamente significantes $(\mathrm{P}<0,05)$ a partir de 2008 para a cobertura da Estratégia Saúde da Família e a partir de 2010 para a cobertura de Equipe de Saúde Bucal (tabela 4).

A relação entre as coberturas de primeira consulta odontológica e ação coletiva de escovação supervisionada segundo o porte populacional não se mostraram estatisticamente significantes. Com relação ao IDH-M, esses indicadores apresentaram correlação positiva estatisticamente significante apenas nos anos de 2001 a 2005 para $1^{\text {a }}$ consulta e no ano de 2006 para a ação coletiva de escovação supervisionada (tabela 5).

Tabela 4- Cobertura média de Equipe da Saúde da Família, Equipe de Saúde Bucal, segundo Índice de Desenvolvimento Humano Municipal no período de 2001-2014, nos Municípios Baianos.

\begin{tabular}{|c|c|c|c|c|c|c|}
\hline \multirow{2}{*}{ Ano } & \multicolumn{4}{|c|}{ IDH-M } & \multirow{2}{*}{\multicolumn{2}{|c|}{$\mathrm{P}$ valor }} \\
\hline & & $0,70-0,79$ & $0,55-0,69$ & $\leq 0,54$ & & \\
\hline \multirow{3}{*}{2001} & & & & & ESF & $\mathrm{ESB}$ \\
\hline & $\mathrm{N}$ & 8 & 362 & 47 & \multirow{3}{*}{0,37} & \multirow{3}{*}{0,33} \\
\hline & ESF & 18,9 & 17,9 & 11,2 & & \\
\hline \multirow{3}{*}{2002} & ESB & 7 & 2,7 & 1,5 & & \\
\hline & $\mathrm{N}$ & 8 & 362 & 47 & \multirow{3}{*}{0,51} & \multirow{3}{*}{0,66} \\
\hline & ESF & 21,6 & 26,9 & 20,5 & & \\
\hline \multirow{3}{*}{2003} & ESB & 7,4 & 7 & 4,9 & & \\
\hline & $\mathrm{N}$ & 8 & 362 & 47 & \multirow[b]{2}{*}{0,89} & \multirow{2}{*}{0,61} \\
\hline & ESF & 26,2 & 32,9 & 32,9 & & \\
\hline \multirow{2}{*}{2004} & ESB & 11 & 11,7 & 8,4 & \multirow{3}{*}{0,75} & \multirow{3}{*}{0,43} \\
\hline & $\begin{array}{r}\mathrm{N} \\
\mathrm{FSF}\end{array}$ & 8 & 362 & 47 & & \\
\hline \multirow{3}{*}{2005} & $\begin{array}{l}\text { ESF } \\
\text { ESB }\end{array}$ & $\begin{array}{l}32,6 \\
20,1\end{array}$ & $\begin{array}{l}38,5 \\
17,2\end{array}$ & $\begin{array}{r}42,2 \\
12\end{array}$ & & \\
\hline & $\mathrm{N}$ & 8 & 362 & 47 & \multirow{3}{*}{0,21} & \multirow{3}{*}{0,25} \\
\hline & ESF & 35,4 & 60 & 60,4 & & \\
\hline \multirow{3}{*}{2006} & ESB & 21,2 & 31,6 & 24,1 & & \\
\hline & $\mathrm{N}$ & 8 & 362 & 47 & \multirow[b]{2}{*}{0,08} & \multirow[b]{2}{*}{0,25} \\
\hline & ESF & 38,7 & 69 & 70,9 & & \\
\hline \multirow{2}{*}{2007} & ESB & 26,2 & 43,6 & 37,4 & \multirow{2}{*}{0,12} & \multirow{3}{*}{0,11} \\
\hline & $\begin{array}{r}\mathrm{N} \\
\text { ESF }\end{array}$ & $\begin{array}{r}8 \\
40.3\end{array}$ & $\begin{array}{r}362 \\
67\end{array}$ & $\begin{array}{r}47 \\
63.6\end{array}$ & & \\
\hline \multirow{3}{*}{2008} & ESB & 25,8 & 39 & 28,5 & & \\
\hline & $\mathrm{N}$ & 8 & 362 & 47 & \multirow[b]{2}{*}{0,03} & \multirow{3}{*}{0,17} \\
\hline & ESF & 41 & 73 & 73,6 & & \\
\hline & ESB & 29,4 & 46,4 & 39,3 & & \\
\hline 2009 & $\mathrm{~N}$ & 8 & 362 & 47 & & \\
\hline & ESF & 40,2 & 77,5 & 76,5 & 0,01 & 0,07 \\
\hline & ESB & 29,8 & 53,2 & 45,2 & & \\
\hline 2010 & $\mathrm{~N}$ & 8 & 362 & 47 & & \\
\hline & ESF & 39 & 84,2 & 87,3 & 0,00 & 0,03 \\
\hline & ESB & 28 & 59,4 & 55,1 & & \\
\hline 2011 & $\mathrm{~N}$ & 8 & 362 & 47 & & \\
\hline & ESF & 43,4 & 87,7 & 88,7 & 0,00 & 0,01 \\
\hline & ESB & 29,1 & 63,4 & 56,5 & & \\
\hline 2012 & $\mathrm{~N}$ & 8 & 362 & 47 & & \\
\hline & ESF & 44,7 & 89,9 & 89,1 & 0,00 & 0,02 \\
\hline & ESB & 31,3 & 65 & 59,4 & & \\
\hline
\end{tabular}




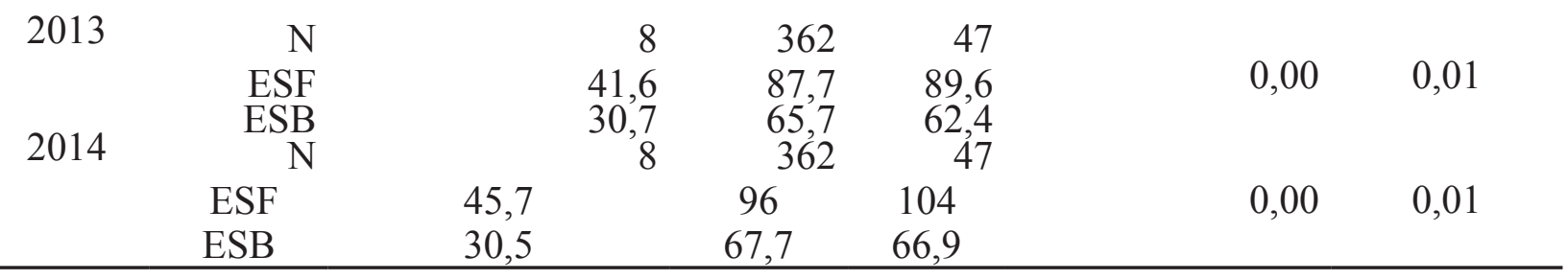

Tabela 5 - Cobertura média de $1^{\text {a }}$ Consulta Odontológica e Escovação Supervisionada segundo Índice de Desenvolvimento Humano Municipal no período de 2001-2014, nos Municípios Baianos.

\begin{tabular}{|c|c|c|c|c|c|c|}
\hline \multirow[b]{3}{*}{ Ano } & & \multicolumn{3}{|c|}{ IDH-M } & \multirow{2}{*}{\multicolumn{2}{|c|}{$P$ valor }} \\
\hline & & \multirow[t]{2}{*}{$0,70-0,79$} & \multirow[t]{2}{*}{$0,55-0,69$} & \multirow[t]{2}{*}{$\leq 0,54$} & & \\
\hline & & & & & $\begin{array}{c}\text { Média } \\
1^{\text {a }} \\
\text { consulta }\end{array}$ & $\begin{array}{c}\text { Média } \\
\text { Escovação }\end{array}$ \\
\hline \multirow[t]{4}{*}{2001} & $\mathrm{~N}$ & 8 & 283 & 35 & \multirow{5}{*}{0,02} & \multirow{5}{*}{0,61} \\
\hline & $1^{\text {a }}$ Consulta & 15 & 9,91 & 4,64 & & \\
\hline & $\mathrm{N}$ & 8 & 168 & 19 & & \\
\hline & Escovação & 0,01 & 0,01 & 0,01 & & \\
\hline \multirow[t]{2}{*}{2002} & $\mathrm{~N}$ & 8 & 296 & 40 & & \\
\hline & $1^{\text {a }}$ Consulta & 17,84 & 11,24 & 6,24 & \multirow{3}{*}{0,01} & \multirow{3}{*}{0,59} \\
\hline \multirow[b]{3}{*}{2003} & $\mathrm{~N}$ & 8 & 209 & 28 & & \\
\hline & $\begin{array}{r}\text { Escovação } \\
\text { Supervisionada }\end{array}$ & 0,01 & 0,03 & 0,03 & & \\
\hline & $\mathrm{N}$ & 8 & 307 & 42 & \multirow{4}{*}{0,04} & \multirow{4}{*}{0,41} \\
\hline \multirow[b]{4}{*}{2004} & $1^{\text {a }}$ Consulta & 16,26 & 12,54 & 7,31 & & \\
\hline & Escovação & 7 & 219 & 30 & & \\
\hline & Supervisionada & 0,02 & 0,03 & 0,02 & & \\
\hline & $\mathrm{N}$ & 8 & 314 & 39 & \multirow{4}{*}{0,02} & \multirow{4}{*}{0,56} \\
\hline \multirow[b]{4}{*}{2005} & $1^{\text {a }}$ Consulta & 17,32 & 12,96 & 7,05 & & \\
\hline & $\stackrel{\mathrm{N}}{\mathrm{F}}$ & 8 & 212 & 27 & & \\
\hline & $\begin{array}{r}\text { Escovaçao } \\
\text { Supervisionada }\end{array}$ & 0,02 & 0,03 & 0,02 & & \\
\hline & $\mathrm{N}$ & 8 & 330 & 39 & \multirow{4}{*}{0,01} & \multirow{4}{*}{0,54} \\
\hline & $1^{\text {a }}$ Consulta & 15,96 & 13,98 & 7,19 & & \\
\hline \multirow[b]{3}{*}{2006} & $\mathrm{~N}$ & 8 & 234 & 31 & & \\
\hline & $\begin{array}{r}\text { Escovação } \\
\text { Supervisionada }\end{array}$ & 0,03 & 0,02 & 0,01 & & \\
\hline & $\mathrm{N}$ & 8 & 332 & 40 & \multirow{3}{*}{0,08} & \multirow{3}{*}{0,00} \\
\hline \multirow{6}{*}{2007} & $1^{\mathrm{a}}$ Consulta & $\begin{array}{r}21,91 \\
8\end{array}$ & $\begin{array}{r}18,18 \\
362\end{array}$ & $\begin{array}{r}10,11 \\
47\end{array}$ & & \\
\hline & $\begin{array}{r}\text { Escovação } \\
\text { Supervisionada }\end{array}$ & 0,04 & 0,01 & 0 & & \\
\hline & $\mathrm{N}$ & 8 & 332 & 44 & \multirow{4}{*}{0,06} & \multirow{4}{*}{0,88} \\
\hline & $1^{\text {a }}$ Consulta & 19,82 & 17,39 & 11,54 & & \\
\hline & $\mathrm{N}$ & 7 & 174 & 13 & & \\
\hline & $\begin{array}{r}\text { Escovação } \\
\text { Supervisionada }\end{array}$ & 0,02 & 0,02 & 0,02 & & \\
\hline
\end{tabular}


2008

2009

2010

2011

2012

2013

2014

$1^{\text {a }}$ Consulta

Escovação

Supervisionada

$\mathrm{N}$

$1^{\text {a Consulta }}$

$\mathrm{N}$

Escovação

Supervisionada

$\mathrm{N}$

$1^{\text {a Consulta }}$

$\mathrm{N}$

Escovação

Supervisionada

$1^{a}$ Consulta

$\mathrm{N}$

Escovação

Supervisionada

$\mathrm{N}$

$1^{\text {a Consulta }}$

$\mathrm{N}$

Escovação

Supervisionada

$\mathrm{N}$

$1^{\text {a }}$ Consulta

N

Escovação

Supervisionada $\begin{array}{lll}8 & 331 & 44\end{array}$

$12,4 \quad 20,04 \quad 8,59$

8 247

27

0,58

0,11

$0,01 \quad 0,02 \quad 0,01$

$8 \quad 342 \quad 42$

$8,55 \quad 18,3 \quad 9,52$

$\begin{array}{lll}8 & 287 & 33\end{array}$

0,27

0,34

$0,01 \quad 0,02 \quad 0,01$

$\begin{array}{lll}8 & 343 & 43\end{array}$

$7,24 \quad 17,85 \quad 22,81$

35

0,49

0,61

$0,01 \quad 0,02 \quad 0,01$

$8 \quad 344$

41

15

35

0,75

0,12

8299

0,07

$\begin{array}{lll}8 & 342 & 41\end{array}$

$19,9 \quad 63,4 \quad 20,9$

8283

34

0,90

0,82

0,01

$\begin{array}{lll}8 & 350 & 42\end{array}$

$5,65 \quad 26,97 \quad 10,45$

$8 \quad 287$

35

0,47

0,76

$0,01 \quad 0,01 \quad 0,01$

$\begin{array}{llll}\mathrm{N} & 8 & 349 & 44\end{array}$

$1^{\text {a } \text { Consulta } \quad 7,05 \quad 23,01}$

$\mathrm{N} \quad 8 \quad 282$

8,29

38

0,52

0,58

0,01

0,01

0,01

\section{DISCUSSÃO}

De modo geral, verificou-se uma tendência de crescimento da produção ambulatorial a partir do ano de 2001, a partir dos indicadores de serviços de saúde bucal: cobertura de $1^{\text {a }}$ consulta odontológica, cobertura de ação coletiva de escovação supervisionada, média de procedimentos de ações básicas (2001-2007), média de procedimentos especializados (2001-2007) (tabela 1). Esse aumento acompanhou a ampliação da cobertura da Estratégia Saúde da Família e das Equipes de Saúde Bucal, de modo que, provavelmente, esteve vinculado ao incentivo financeiro para 
inserção das Equipes de Saúde Bucal na Estratégia Saúde da Família (ESB/ESF), estabelecido pelo Ministério da Saúde em dezembro de 2000, através da Portaria ${ }^{\circ} 1.444$, que além de reestruturar as ações de saúde, buscou promover a expansão e reorganização das atividades de saúde bucal, pautadas nos princípios do Sistema Único de Saúde (SUS) ${ }^{10}$. Vale destacar que a partir de 2005 há um crescimento mais acentuado, provavelmente reflexo dos investimentos decorrentes da Política Nacional de Saúde Bucal².

A inserção do cirurgião-dentista na Estratégia Saúde da Família configurou-se como ampliação da rede de serviços de saúde bucal ${ }^{18,19}$. Isso ocorreu, inclusive, devido à implementação desse repasse financeiro, que implicou em um aumento de mais de dez vezes dos recursos federais destinados à saúde bucal entre 2003 e $2008^{19}$. Em janeiro de 2002, pouco mais de um ano após a inclusão da saúde bucal na ESF, o país contava com 2.467 Equipes de Saúde Bucal em 1.396 municípios ${ }^{20}$. Crescimento importante, mas relativamente pequeno quando comparado com o total de ESF $(13.661)^{20}$ e de municípios com ESF (3.740) ${ }^{20}$ naquele momento. No estado do Rio de Janeiro, entre 2004 e 2010, houve significativo aumento na implantação de ESB/ESF e Centros de Espacialidades Odontológicas (CEO), correspondendo também a um aumento na provisão de serviços odontológicos e contribuindo para melhora dos indicadores de serviços de saúde bucal (ações odontológicas básicas, primeira consulta programática, procedimentos coletivos, preventivos individuais e cirúrgicos) ${ }^{15}$. Em 2011, o estado da Bahia já tinha 409 municípios com equipes de saúde bucal, em um total de 1.911 equipes, sendo 99,4\% na modalidade I (cirurgião-dentista e auxiliar de saúde bucal) ${ }^{21}$, evidenciando que a Política Nacional de Saúde Bucal não tem sido suficiente para incentivar a inclusão de técnicos em saúde bucal à Estratégia Saúde da Família, o que poderia contribuir ainda mais no avanço da cobertura populacional ${ }^{22}$.

Na capital do estado, em 2004, observava-se um quadro de baixa cobertura de equipes de saúde bucal na ESF, bem como baixo acesso da população aos serviços e ações de saúde bucal ${ }^{23}$. A rede de atenção à saúde bucal do município de Salvador era insuficiente para o porte populacional entre 2004 e 2007, como também era quase inexistente a oferta de serviços especializados em odontologia $^{23}$. Essa escassez de serviços especializados em saúde bucal, é uma realidade importante na Bahia, o que pode ser observado pelas médias de produção ambulatorial de ações especializadas até 2007 (Tabela 3), bem como pela quantidade, distribuição e tipo de Centros de Especialidades Odontológicas no estado: em 2019, são 79 CEO, sendo 40,5\% tipo 1 (até 3 cadeiras), 49,4\% tipo 2 (até 6 cadeiras) e apenas 10,1\% tipo 3 (sete ou mais cadeiras), distribuídos em 72 municípios $(17,26 \% \text { do total de municípios do estado })^{24}$.

Estudo realizado com 185 municípios do estado de Pernambuco observou que, em municípios de menor porte, eram priorizados procedimentos clínicos, mostrando uma baixa valorização das ações de promoção da saúde ${ }^{18}$. No caso da Bahia, essa priorização de ações clínicas em detrimento das ações de prevenção de doenças e promoção da saúde, foi evidenciada nos municípios com maior IDH-M (Tabelas 4 e 5), não tendo sido identificadas diferenças significantes com relação ao porte populacional.

ISSN 1982-8829 Tempus, actas de saúde colet, Brasília, 13(3), 25-42, set, 2019. Epub Jul/2020 
O fato da priorização de atividades clínicas acontecer em diferentes contextos, leva-nos a refletir sobre dois aspectos: o habitus, no sentido que lhe é dado por Bourdieu ${ }^{25}$, de princípio gerador de disposições, e a formação profissional, enquanto inculcadora de princípios e formadora de um habitus de classe. Nesse sentido, alguns profissionais apresentam limites para incorporar tecnologias para a promoção da saúde, a prevenção de doenças e atendimento aos princípios do SUS, que contribuiriam para uma efetiva mudança no modelo assistencial ${ }^{18}$. Faz-se então necessária a reflexão sobre a formação profissional e sua adequação ao SUS. Profissionais consideram que sua formação foi insuficiente para a atuação na Estratégia Saúde da Família ${ }^{26}$, mas a incorporação de estágios na atenção primária do SUS tem possibilitado um olhar ampliado sobre a saúde, o acolhimento, o trabalho em equipe ${ }^{27}$ e uma melhor compreensão da produção do cuidado em saúde $^{28}$ na formação do cirurgião-dentista.

Ainda que a prática liberal se apresente como imagem objetivo para muitos dentistas, a odontologia de mercado tem passado por um processo importante de precarização ${ }^{29}$. Nesse sentido, a ampliação do mercado de trabalho na esfera pública a partir dos anos 2000, tem revelado a Estratégia Saúde da Família como um espaço importante de inserção do cirurgião-dentista e induzido a incorporação de novas perspectivas e abordagens na formação profissional.

Desde a sua inclusão na estratégia saúde da família, a saúde bucal sempre esteve entre os componentes monitorados no Pacto de Indicadores da Atenção Básica, através de indicadores de cobertura das equipes e serviços de saúde bucal. Essa iniciativa contribuiu para a indução do monitoramento de indicadores pelo nível municipal, levando a uma melhora significativa no número de municípios com registro de informações de procedimentos no SIA-SUS, como observado no presente estudo, bem como na qualidade da informação.

A análise de alguns indicadores de saúde bucal do Estado de Pernambuco evidenciou que quanto maior a cobertura de ESB/ESF, na relação de 1 ESB para cada 1 ESF, mais favoráveis eram os indicadores de primeira consulta odontológica, escovação supervisionada, média de procedimentos odontológicos individuais e proporção de exodontias ${ }^{18}$. A não inclusão dessa informação para análise do estado da Bahia configura um limite deste trabalho.

Os indicadores de saúde constituem uma importante ferramenta para avalição dos recursos destinados a atenção da saúde, uma vez que, nos possibilitam mensurar o impacto da aplicação destes recursos para a população. Alguns estudos mostram que os indicadores de saúde bucal são instrumentos importantes na avalição dos recursos destinados a essa área na atenção básica, contudo, os indicadores adotados atualmente na área de saúde bucal têm um forte viés produtivista, ainda que seja possível caracterizar o modelo de atenção a partir da sua análise ${ }^{7}$. Faz-se necessário continuar monitorando a implementação da política de saúde bucal através dos indicadores propostos para reorientação da política no sentido de melhor atender às necessidades de saúde da população, mas também pensar novos indicadores que sejam capazes de abranger outras dimensões para além da produção ambulatorial.

Tempus, actas de saúde colet, Brasília, 13(3), 25-42, set, 2019. Epub Jul/2020 ISSN 1982-8829 


\section{CONCLUSÃO}

Houve aumento nos os indicadores de produção ambulatorial de saúde bucal analisados, apresentando oscilação em alguns períodos. Observou-se aumento expressivo das coberturas da Equipe Saúde da Família (ESF) e de Equipe de Saúde Bucal (ESB), no período de 2001 a 2014 , bem como da média de ações básicas.

Indicadores como cobertura de primeira consulta odontológica, média de escovação supervisionada e média de ações especializadas apresentaram crescimento em alguns períodos e decréscimo em outros. Quanto menor o porte populacional, maior foi a cobertura da ESF e de ESB. Com relação ao IDH-M, observou-se que quanto menor, maior é a cobertura de ESF e ESB.

Os indicadores fazem parte das conquistas vinculadas a oportunidade de acesso à atenção básica; alcançada por meio de políticas públicas, que tem como objetivos expandir o acesso à população, tornando visíveis os direitos previstos pelo Sistema Único de Saúde. A avaliação dos indicadores de saúde bucal dos municípios baianos no período estudado, contribuem para uma avaliação não apenas da política de saúde bucal vigente, mas, sobretudo para auxiliar gestores no planejamento dos serviços de saúde. Releva-se importante, ainda, o debate sobre a construção de indicadores que possibilitem uma análise para além da produção ambulatorial, com potencial de articulação com dados de levantamentos epidemiológicos e demais dados que apontem para o modelo de atenção à saúde priorizado localmente.

\section{REFERÊNCIAS}

1. Chaves SCL, Moysés SJ. Política e saúde: conceitos básicos e abordagens teóricometodológicas para análise política em saúde bucal. In: Chaves SCL. (Ed.). Política de Saúde no Brasil: teoria e prática. Salvador- Bahia: EDUFBA; 2016. p.13-46.

2. Brasil. Ministério da Saúde. Secretaria de Atenção à Saúde. Departamento de Atenção Básica. Coordenação Nacional de Saúde Bucal. Diretrizes da Política Nacional de Saúde Bucal. Brasília: Ministério da Saúde: 16p. 2004.

3. Fernandes JDKB, et al. Avaliação dos indicadores de saúde bucal no Brasil: tendência evolutiva pró-equidade? Cad Saude Publica. 2016; 32(2):e00021115. Disponível em: < http:// www.scielo.br/scielo.php?script=sci_arttext\&pid=S0102-311X2016000200701\&lng=en>.

4. Sousa MFD, Hamann EM. Programa Saúde da Família no Brasil: uma agenda incompleta? Ciência \& Saúde Coletiva. 2009;14:1325-1335.

5. Paludetto Junior M. Avaliação de desempenho da Política Nacional de Saúde Bucal no período de 2004-2010. 2013. 104p. Dissertação (Mestre). Universidade de Brasília, Brasília.

ISSN 1982-8829 Tempus, actas de saúde colet, Brasília, 13(3), 25-42, set, 2019. Epub Jul/2020 
6. Mendes Júnior FIR, Bandeira MAM, Tajra FS. Percepção dos profissionais quanto à pertinência dos indicadores de saúde bucal em uma metrópole do Nordeste brasileiro. Saúde em Debate. 2015;39:147-158.

7. Barros SGD, Chaves SCL. A utilização do Sistema de Informações Ambulatoriais (SIASUS) como instrumento para caracterização das ações de saúde bucal. Epidemiologia e Serviços de Saúde. 2003; 12:41-51.

8. Sistema de Informações Ambulatoriais.: Ministério da Saúde. Departamento de Informática do SUS (Datasus). 2015.

9. Silva SFD, et al. Análise do avanço das equipes de saúde bucal inseridas na Estratégia Saúde da Família em Pernambuco, região Nordeste, Brasil, 2002 a 2005. Ciência \& Saúde Coletiva. 2011; 16:211-220.

10. Pimentel BV, et al. A utilização dos indicadores de saúde bucal e de desenvolvimento humano no monitoramento da atenção básica nos municípios da região metropolitana de Curitiba-PR. Revista espaço para a saúde. jul-set 2014;15(3):42-52.

11. Cruz DFD, et al. A Linha do Cuidado em Saúde Bucal no Município de João Pessoa: Uma Análise de Indicadores. Pesqui. bras. odontopediatria clín. integr. 2011; 11(02):291-295. Disponível em: < http://revista.uepb.edu.br/index.php/pboci/article/viewFile/1029/677>.

12. Cavalcanti YW, et al. Associação de Indicadores de ProduçãoOdontológica e de Condição Sócio-Sanitária naAtenção Básica de João Pessoa - PB. Rev. bras. ciênc. saúde. 2010; $14(3): 47-52$.

13. Fischer TK, et al. Indicadores de atenção básica em saúde bucal: associação com as condições socioeconômicas, provisão de serviços, fluoretação de águas e a estratégia de saúde da família no Sul do Brasil. Rev Bras Epidemiol. 2010; 13(1):126-138.

14. Zermian TC, et al. Indicadores de desenvolvimento humano e de saúde bucal na atenção básica nos municípios da região metropolitana de Curitiba-PR. RFO UPF. 2014;19(2): 185-192.

15. Scarparo A, et al. Impacto da Política Nacional de Saúde Bucal - Programa Brasil Sorridente - sobre a provisão de serviços odontológicos no Estado do Rio de Janeiro. Cad. saúde colet.2015;23(4):409-415.

16. Pereira CRDS, et al. Impacto da Estratégia Saúde da Família sobre indicadores de saúde 


\section{$41 / /$}

bucal: análise em municípios do Nordeste brasileiro com mais de 100 mil habitantes. Cadernos de Saúde Pública. 2012;28:449-462.

17. Magri LV, et al. Estudo comparativo de indicadores de saúde bucal em município do estado de São Paulo. Saúde em Debate. 2016; 40:144-155.

18. Pimentel FC, et al. Analysis of oral health indicators of Pernambuco: performance of cities according to size population, population enrolled in the Information System for Primary Care and proportion in the Family Health Strategy. Cadernos Saúde Coletiva. 2014;22:54-61.

19. Frazão P, NARVAI PC. Saúde bucal no Sistema Único de Saúde: 20 anos de lutas por uma política pública. Saúde em Debate. 2009; 33(81):64-71.

20. Vieira-da-Silva L, et al. O Programa de Saúde da Família: evolução de sua implantação no Brasil - Relatório final. Instituto de Saúde Coletiva- UFBA / Ministério da Saúde - Secretaria de Políticas de Saúde: Salvador, Bahia. 70p. 2002.

21. Bahia. Secretaria de Saúde do Estado da Bahia. Superintendência de Atenção Integral à Saúde. Diretoria de Atenção Básica. Boletim de Avaliação e Monitoramento da Atenção Básica - Bahia. Salvador, Bahia: Secretaria da Saúde do Estado da Bahia: 4 p. 2017.

22. Aguiar DML, Frazão P. A insuficiência da política pública para inclusão do técnico em saúde bucal na Atenção Primária no Brasil. In: Chaves SCL. (Ed.). Política de saúde bucal: teoria e prática. Salvador, Bahia: EDUFBA, 2016. p.297-318. ISBN 978-85-232-1500-2.

23. Soares CLM, Paim JS. Aspectos críticos para a implementação da política de saúde bucal no Município de Salvador, Bahia, Brasil. Cadernos de Saúde Pública. 2011;27:966-974.

24. Cidades que possuem Centros de Especialidades Odontológicas - CEO Bahia MS/SAS/DAB/Coordenação Geral de Saúde Bucal - CGSB 2019.

25. Bourdieu P. A distinção: crítica social do julgamento. São Paulo, Porto Alegre: Edusp, Zouk, 2008. 560p.

26. Pinheiro FMC, et al. A formação do cirurgião-dentista e a promoção de saúde no PSF. Revista de Odontologia da UNESP. 2008; 37(1):69-77.

27. Grande IMP, et al. Desafios na formação do Cirurgião-Dentista para o SUS. Revista da ABENO. 2016; 16(3):2-6. 
28. Baumgarten A., Toassi RFC. A formação do cirurgião-dentista no Sistema Único de Saúde: a produção do cuidado em saúde. Rev. Bras. Pesq. Saúde.out-dez 2013; 15(4):117-122.

29. Bleicher L. Precarização do trabalho do cirurgião-dentista na cidade de Salvador, Bahia. Revista Baiana de Saúde Pública. 2013;36(3): 668-682.

Artigo apresentado em outubro de 2019 Artigo aprovado em janeiro de 2020 Artigo publicado em julho 2020 